\section{Neuropathischer Schmerz Was hilft, wenn NSAR versagen?}

\author{
Bei Rückenschmerzen mit neuro- \\ pathischer Schmerzkomponente, \\ etwa bei Patienten mit chronischer \\ Lumboischialgie, sind NSAR wirkungs- \\ los, da diese Analgetika nur nozizeptive \\ Schmerzen lindern.
}

Bei neuropathischen Schmerzen oder bei gemischt neuropathisch-nozizeptiven Schmerzen („Mixed Pain”) sind Koanalgetika die Mittel der Wahl.

\section{Antikonvulsiva helfen}

Infrage kommen zum einen Antikonvulsiva wie Gabapentin oder Pregabalin (Lyrica $\left.{ }^{\star}\right)$. Beide Substanzen sind zur Therapie von Patienten mit neuropathischen Schmerzen zugelassen.

\section{Option Antidepressiva}

Zum anderen sind einige Antidepressiva eine bewährte Option bei Patienten mit chronischen (Rücken-) Schmerzen.

\section{Abrechnungs-Tipp Genehmigung}

Patienten mit Rückenschmerzen gehö ren für Allgemeinmediziner zum Praxisalltag. Sind sie durch Wirbelblockaden verursacht, ist die Chirotherapie eine wirkungsvolle Therapie. Diese können Sie nach EBM mit den Ziffern 30200 (Extremitätengelenke) und 30201 (Wirbelsäule) berechnen. Voraussetzung sind der entsprechende Qualifikationsnachweis und die Genehmigung der KV. Diese Genehmigung ermöglicht evtl. den Zugriff auf das QZV Chirotherapie, und sie eröffnet den Zugriff zu dem gesamten Kapitel 30.4 des EBM. Hier könnten die Nr. 30420 (Krankengymnastik, Einzelbehandlung) und die

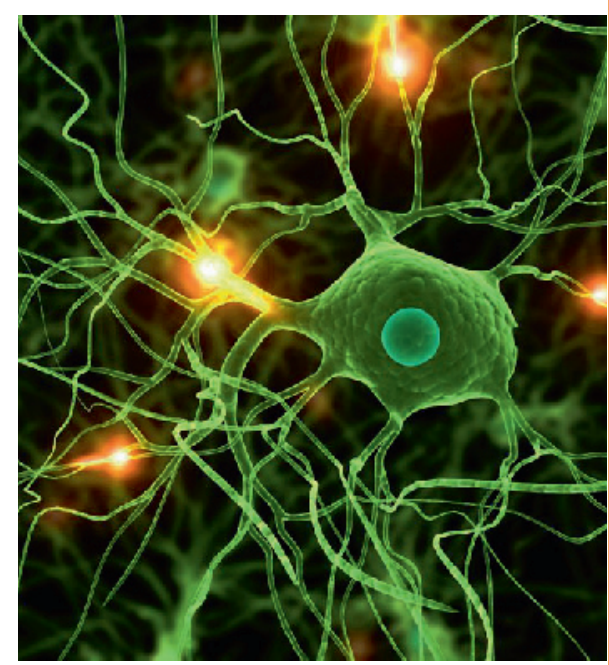

Sind die Nerven am Rückenschmerz beteiligt, kommen NSAR nicht infrage.

$\mathrm{Zu}$ den Antidepressiva, für die auch schmerzlindernde Wirkungen belegt sind, gehören außer den trizyklischen Substanzen auch Mirtazapin, Reboxetin, Venlafaxin und Duloxetin. Dabei ist die analgetische Wirkung früher spürbar als die antidepressive.

(mar)

\section{$>$ Übrigens ...}

... lindert Duloxetin auch chronische Kreuzschmerzen ohne neuropathische Komponente, wie eine Phase-III-Studie mit 401 Betroffenen ergeben hat. Im Vergleich zu Placebo ließ sich eine signifikante Schmerzreduktion erzielen.

J Pain 2010, online first

... wirkt sich eine kognitive Verhaltenstherapie bei Patienten mit subakuten oder chronischen Kreuzschmerzen auch noch nach zwölf Monaten positiv auf den Schmerzverlauf aus. Das hat eine Studie mit fast 600 Patienten aus 56 Hausarztpraxen verdeutlicht. Zwei Drittel der Patienten hatten zusätzlich zum üblichen Beratungsgespräch in den ersten Studienwochen eine kognitive Verhaltenstherapie (sechs jeweils 1,5-stündige Sitzungen) erhalten. Bei $59 \%$ von ihnen hatten sich die Schmerzen und Funktionseinschränkungen langfristig gebessert, in der Gruppe mit alleinigem Beratungsgespräch bei nur 31\%. Lancet 2010, 375:916

\section{Infos im Internet}

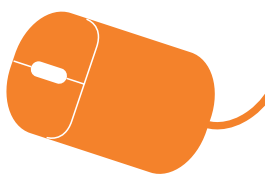

Alle Folgen der Sommer-Akademie finden Sie im Web unter www.aerztezeitung.de

\section{Chirotherapie abrechnen}

Bei Privatpatienten können Sie auch die Untersuchung des Bewegungsapparates (Nr. 7) mit der neurologischen Untersuchung (Nr. 800) kombinieren. Dazu rechnen Sie die Nr. 3306 für die Chirotherapie ab. Bewegungsübungen, Übungsbehandlungen und die paravertebrale Injektionsbehandlung können Sie zusätzlich mit den Nrn. 507, 510 und 267 berechnen. Natürlich vergessen Sie nicht die Beratung nach Nr. 1.

(pes)
Derzeit wird interdisziplinär die Nationale VersorgungsLeitlinie Kreuzschmerz erstellt. Die Konsultationsfassung gibt es unter

w w w.versorgungsleitlinien.de/ themen/kreuzschmerz

Der Deutsche Schmerzkongress 2010 findet vom 06.-09.10.2010 in Mannheim statt. Infos unter

www.schmerzkongress2010.de 